\section{NEURODEGENERATION}

\section{A translational block}

Several events that contribute to cellular dysfunction are involved in Alzheimer's disease pathology, raising the issue of how amyloid $\beta$ $(A \beta)$ peptides can mediate such wide-ranging effects. Now, a recent study may provide a partial explanation by showing that oligomeric $\mathrm{A} \beta_{42}$ blocks the key process of translation (Neuron 75, 824-837).

Song Ok Yoon et al. found that oligomeric $A \beta_{42}$ induces a translational block in cultured neurons by activating AMP-activated protein kinase, which then leads to inhibition of the mammalian target of rapamycin pathway. The translational block leads to an unfolded protein response that activates $\mathrm{C}$-Jun $\mathrm{N}$-terminal kinase 3 (JNK3). Activated JNK3 then phosphorylates the amyloid precursor protein (APP) at a particular site (Thr668) that promotes APP endocytosis and proteolytic processing of APP to $A \beta$ peptides.

The authors found that JNK3 activity was increased in cortical samples from patients with Alzheimer's disease and in brains from mice modeling a form of familial Alzheimer's disease (FAD). Deletion of JNK3 in FAD mice decreased the amounts of $A \beta_{42}$ and the number of plaques in their brains compared with FAD mice with active JNK3. In addition, JNK3 deletion partially increased neuronal numbers and improved neuronal phenotypes in the FAD mice.

The authors suggest that JNK3 activation is necessary for the operation of a positive feedback loop that leads to the continual production of $A \beta_{42}$; thus, JNK3 could be a potential therapeutic target for Alzheimer's disease.-MS

\section{DIABETES}

\section{Dedifferentiation, not death}

A long-standing question in the diabetes field is whether pancreatic beta cell failure is a result of reduced numbers of beta cells or loss of their function. A new study by Chutima Talchai et al. (Cel/ 150, 1223-1234) may help resolve this issue.

FoxO1 is a key transcription factor that integrates stress responses and regulation of beta cell mass. The authors examined mice that had a beta cell-specific deletion of FoxO1 and found that, when the mice were exposed to physiological stress, they showed hyperglycemia and reduced beta cell mass. Using lineage-tracing studies, the authors showed

IMMUNOLOGY

\title{
Commensals under attack
}

$T$ cells specific for commensals expand and differentiate into memory cells as a result of a parasitic infection in the gastrointestinal tract, according to a new study (Science doi:10.1126/ science.1220961).

Immune tolerance to commensals in the gut is kept in check by numerous adaptive and innate immune mechanisms, preventing unwanted proinflammatory responses and mucosal damage. Whether these immune mechanisms are breached by a pathogenic infection remains unclear.

Yasmine Belkaid and her colleagues found that infection with the parasite Toxoplasma gondii induced the expansion of $\mathrm{CD}^{+}{ }^{+} \mathrm{T}$ cells specific to commensal-derived flagellin. These $T$ cells adopted a $T$ helper type $1\left(T_{H} 1\right)$ phenotype and secreted interferon- $\gamma$. Although this pool of $\mathrm{T}$ cells contracted over time, a small population formed memory T cells that persisted up to $\mathbf{2 4 0}$ days after infection and could be activated upon infectious challenge. Although it remains unclear whether these commensal-specific memory T cells directly contribute to epithelial damage and susceptibility to inflammatory bowel disease, these findings suggest that tolerance to commensal bacteria can be impaired by gastrointestinal infection. -KDS

that this decrease in cell mass resulted from beta cell dedifferentiation rather than from cell death. Importantly, Talchai et al. showed that two other mouse models of type 2 diabetes show progressive loss of FoxO1 expression during diabetes development and also beta cell differentiation, suggesting that dedifferentiation is a shared mechanism.

Investigation of whether beta cell dedifferentiation also occurs in human diabetes will be needed, but the authors suggest that if so, a revised paradigm for therapeutic strategies will be required-promoting beta cell differentiation rather than replication.-MS

\section{CANCER}

\section{Finding fusions}

Two recent studies identify new gene fusions in solid tumors in humans and provide clues into their role in tumorigenesis (Nature $\mathbf{4 8 8}$, 660-664; Science 337, 1231-1235).

Somasekar Seshagiri et al. identified recurrent gene fusions involving R-spondin family members ( $R S P O 2$ and RSPO3) in 10\% of colon tumors. They characterized EIF3E-RSPO2 and PTPRK-RSPO3 gene fusions and found that colon tumor samples bearing R-spondin fusions showed increased expression of RSPO2 and $\mathrm{RSPO} 3$ compared with colon tumors lacking fusions. R-spondins potentiate canonical Wnt signaling, and consistent with this, the authors found that RSPO fusion constructs led to Wnt pathway activation in a human colon cancer cell line and that tumor samples with RSPO fusions showed upregulation of Wnt signaling. RSPO fusions were mutually exclusive with mutations in the Wnt pathway genes $A P C$ or CTNNB1 in colon cancers, suggesting that in the absence of these mutations the RSPO fusions may have a role in activating Wnt signaling and tumorigenesis.

In another study, Devendra Singh et al. identified a small set of glioblastoma multiforme $(3.1 \%)$ with recurrent, oncogenic gene fusions involving the tyrosine kinase-encoding domain of a fibroblast growth factor gene (FGFR1 or FGFR3) joined to a transforming acidic coiled-coil gene (TACC1 or TACC3, respectively). The fusion proteins have constitutive kinase activity and lead to mitotic delay and aneuploidy. Lentiviruses expressing one of the FGFR-TACC fusion proteins could transform cultured fibroblasts or astrocytes, and injection of FGFR-TACC transduced astrocytes into immunodeficient mice led to the formation of invasive, glioma-like tumors. Treatment of mice bearing FGFR3-TACC3 glioma xenografts with an FGFR inhibitor inhibited tumor growth and prolonged survival compared with untreated mice. Thus, FGFR inhibitors may have potential clinical application in patients with GBM bearing FGFR-TACC fusions.-MS

Written by Kevin Da Silva and Meera Swami 